\title{
Magnetic field at the inner disk edge
}

\author{
Moira Jardine ${ }^{1}$, Scott G. Gregory ${ }^{1}$ \\ and Jean-François Donati ${ }^{2}$ \\ ${ }^{1}$ SUPA, School of Physics and Astronomy, North Haugh, St Andrews, UK, KY16 9SS \\ email: mmj@st-andrews.ac.uk, sg64@st-andrews.ac.uk \\ ${ }^{2}$ Laboratoire dAstrophysique, Observatoire Midi-Pyrénées, 14 Av. E. Belin, F-31400 Toulouse, \\ France \\ email: donati@ast.obs-mip.fr
}

\begin{abstract}
Our present understanding of the coronal structure of T Tauri stars is fragmentary and observations in different wavelength regimes often appear to give contradictory results. Xray data suggest the presence of magnetic loops on a variety of scales, from compact loops of size less than a stellar radius, up to very large loops of up to 10 stellar radii which may connect to the disk. While some stars show a clear rotational modulation in X-rays, implying distinct bright and dark regions, many do not. This picture is complicated by the accretion process itself, which also contributes to the X-ray emission. The location of the inner edge of the accretion disk and the nature of the magnetic field there are still hotly-contested issues. Accretion indicators often suggest the presence of discrete accretion funnels. This has implications for the structure of the corona, as does the presence of an outflowing wind. All of these factors are linked to the structure of the magnetic field, which we are now beginning to unravel through Zeeman-Doppler imaging. In this review I will describe the present state of our understanding of the magnetic structure of $\mathrm{T}$ Tauri coronae and the impact this has during such an early evolutionary stage.
\end{abstract}

Keywords. Stars:coronae

\section{Introduction}

$\mathrm{T}$ Tauri stars represent a fascinating phase of stellar evolution. It is during this period, when stars of intermediate to low mass contract onto the main sequence, that planets may form in the disks around these stars. Two outstanding questions remain however about this phase of stellar evolution. The first is the rate at which material can accrete from this disk onto the star. This influences the lifetime of the disk and so sets the challengingly brief timescale for planet formation. The second question is the mechanism by which these stars lose angular momentum. Most $\mathrm{T}$ Tauri stars are fairly slow rotators, despite their contraction and the possible gain in angular momentum associated with accretion of material from a surrounding disk.

The nature of the stellar magnetic field is central to both of these questions. The stellar magnetic field disrupts the inner edge of the accretion disk and channels the accreting material onto the star in accretion funnels. This process not only governs the torques that are exchanged between the disk and the star but also, by determining the inner extent of the accretion disk, it may influence the minimum orbital radius of exoplanets (Romanova \& Lovelace 2006). Only a certain fraction of the stellar field, however, intersects the accretion disk and contributes to this process. Some stellar field lines may be closed and never intersect the disk. These field lines will contribute to the X-ray emission of the star and may provide the "coronal component" of the X-ray spectrum. The remaining field lines are open and can carry the stellar wind, thus allowing the star to lose angular momentum. 

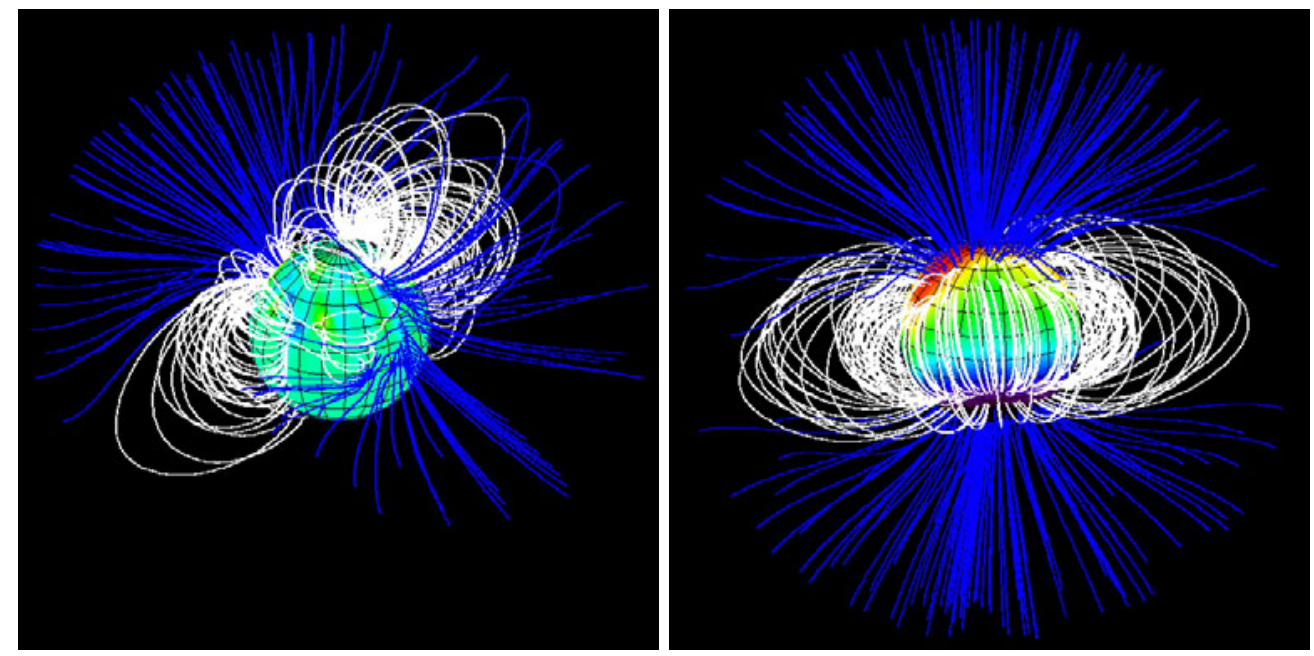

Figure 1. Field structures for the marginally pre-main sequence $1 \mathrm{M}_{\odot}$ star AB Dor (left) and the fully-convective main sequence $0.3 \mathrm{M}_{\odot}$ star V374 Peg (right) extrapolated from Zeeman-Doppler surface magnetic maps (Donati et al. 1999; Donati et al. 2006). White denotes closed field lines, while blue denotes open field lines.

\section{Observational indicators of magnetic structure}

The last few years have seen great advances in our knowledge of the surface magnetic fields of $\mathrm{T}$ Tauri stars. Zeeman-Broadening measurements can now be used to measure mean fields on the stellar surface (Johns-Krull et al. 2004; Valenti \& Johns-Krull 2004; Yang et al. 2005). This gives distributions of local field strengths on the stellar surface that may be as high as $6 \mathrm{kG}$. The results are similar on both accreting and non-accreting $\mathrm{T}$ Tauri stars and show little correlation with either the stellar rotation rate or Rossby number.

A complementary approach is to use circular polarisation measurements either in photospheric lines (presumably formed across the whole stellar surface) or the HeI $5876 \AA$ emission line believed to form in the accretion shock (Yang et al. 2007). Polarisation measurements taken at a range of rotation phases can then be used to provide information on the structure of the magnetic field both across the surface and in the accretion shock. The low circular polarisation measured in photospheric lines rules out the presence of a global dipole, suggesting perhaps a complex field that may be locally very intense, but which is organised into small enough scales that the individual polarisations cancel out. In comparison, the strong, rotationally modulated polarisation in the Helium emission lines suggests that the field associated with accretion is much simpler with few reversals in sign. This is no doubt to be expected, since it is the largest-scale field lines that will interact with the disk and these are likely to have the simplest structure.

The nature of the star-disk interaction will clearly depend on the structure of the whole coronal field, and in particular the degree to which the complexity that seems apparent at the stellar surface may have died away by the time the coronal field reaches the inner edge of the disk. This is illustrated in Fig. 1 which shows, for two very different stars, coronal field structures extrapolated from Zeeman-Doppler maps of the surface field. This is done using the Potential Field Source Surface method developed many years ago for modelling the solar corona (Altschuler \& Newkirk Jr. 1969). This assumes that at some height above the stellar surface (known as the source surface) the outward pressure of the hot coronal gas forces the magnetic loops open and allows the gas to escape to 


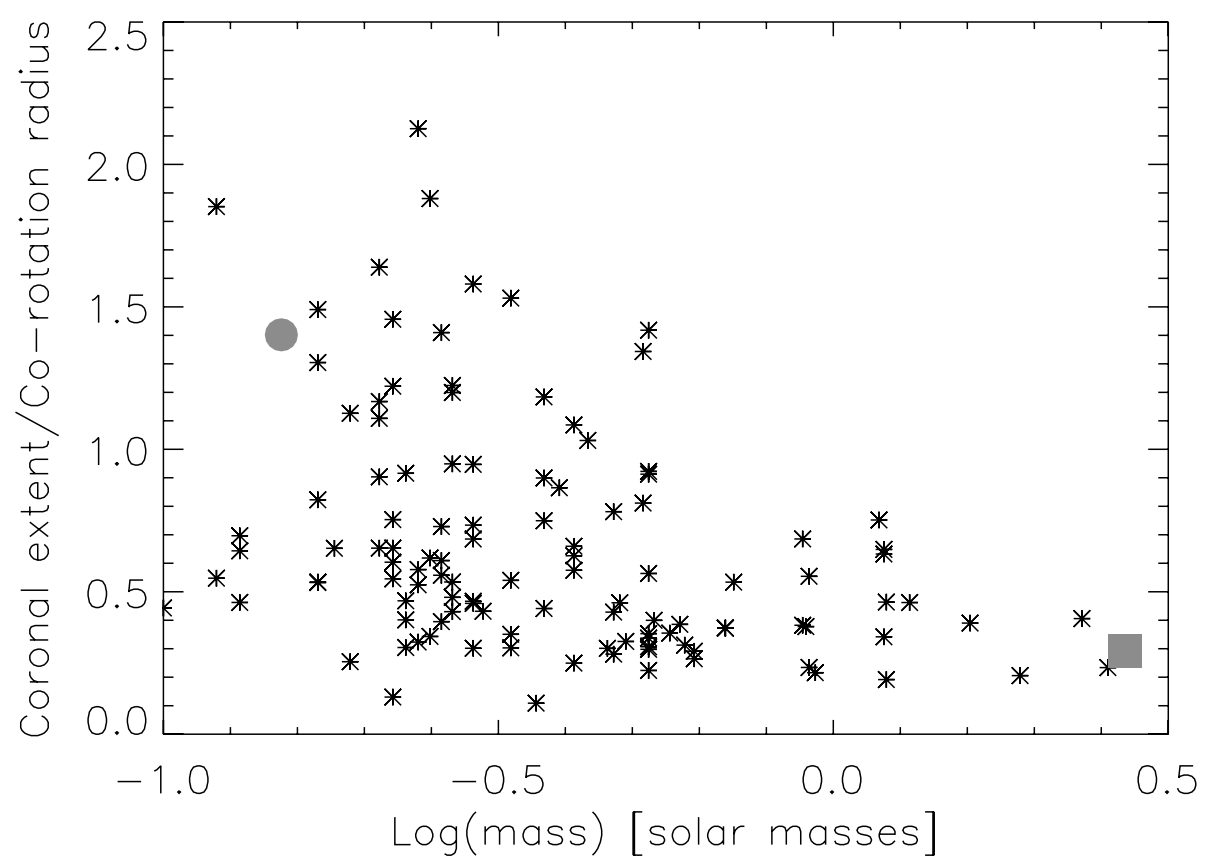

Figure 2. Calculated coronal extent (in units of the Keplerian co-rotation radius) as a function of stellar mass for stars in the COUP sample. Two stars (marked with a circle and square) have been selected as examples. Their coronal structure is shown more fully in Fig. 3. Taken from Jardine et al. (2006).

form the stellar wind. Inside the source surface, the field is potential and so along each field line the gas settles into hydrostatic balance (Jardine et al. 2002a,b). As can be seen from Fig. 1, the magnetic fields on stars with different internal structures can be very different. The higher-mass star (AB Dor) has a radiative core and a dynamo that produces a field that is complex at the stellar surface although on the largest scale it resembles a tilted dipole. The lower-mass star (V374 Peg), is fully convective and has a field structure that resembles an aligned dipole. On the largest scales, such fields are both simple and would interact with an accretion disk (if one were present) to produce perhaps only one accretion funnel in each hemisphere. An accretion disk that penetrated closer to the star, however, would intersect a much more complex field in the first case, possibly producing many more accretion funnels.

These results suggest that the growth of the radiative core in $\mathrm{T}$ Tauri stars may be associated with the onset of a different type of dynamo activity and hence a different coronal field structure. Some indication of this may be apparent in the COUP sample of stars. Those that are expected to have a radiative core appear to have a lower X-ray luminosity than their fully-convective counterparts (Rebull et al. 2006).

The nature of the star-disk interaction is also likely to be influenced by the coronal structure. Recent results by Jardine et al. (2006) suggest that the nature of the star-disk interaction may be fundamentally different in higher and lower mass stars. If we consider pre-main sequence stars of progressively lower mass, their pressure scale heights $\Lambda$ will increase (since for a polytrope $R \propto M_{*}^{-1 / 3}$ and so $\Lambda \propto M_{*}^{-5 / 3}$ ) while their co-rotation radii will decrease. Thus the lower the stellar mass, the more likely it is that the corotation radius will be within the corona (see Figs. 2 and 3). If a disk is present, this 

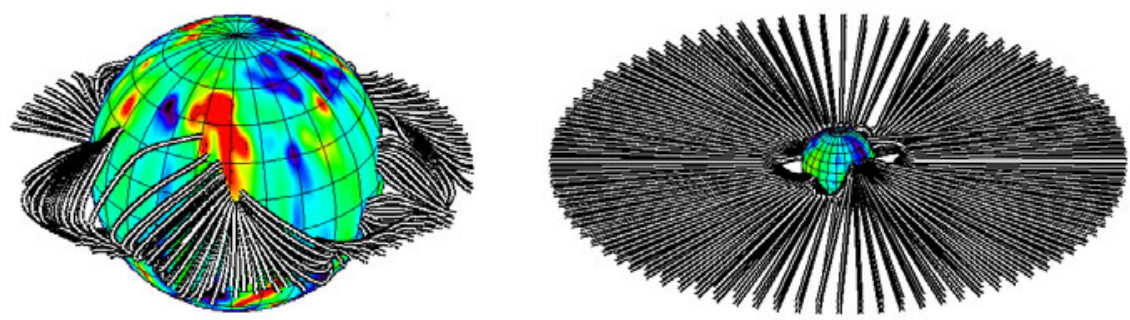

Figure 3. Calculated coronal structure for the lower-mass (left) and the higher mass (right) T Tauri star shown in Fig. 2. For the lower mass star, the natural extent of the corona is greater than the co-rotation radius, and so the disk strips off the outer parts of the corona, reducing the available X-ray emitting volume. For the higher mass star, the corona is well within the co-rotation radius and so the field lines that interact with the disk are the open ones. Taken from Jardine et al. (2006).

may mean that the disk strips off the outer edge of the corona, but even in the absence of a disk, centrifugal effects may do the same job. As Fig. 4 shows, this not only explains the drop in X-ray emission measures towards lower masses seen in the COUP dataset (Getman et al. 2005; Preibisch et al. 2005), but it also suggests that for the higher-mass stars, whose coronae may not extend as far as the co-rotation radius, any disk that exists at the co-rotation radius may interact not with the closed X-ray emitting field lines of the star but rather with the open, wind-bearing lines (see Fig. 3). A further calculation of the associated accretion rates as a function of stellar mass also reproduced the accepted relationship (Gregory et al. 2006b).

\section{Implications for angular momentum loss}

The importance of the open field lines (i.e. those with one footpoint on the star and the other either attached to the disk, or to the interstellar field) has become apparent from several independent lines of research. It has also emerged out of MHD modelling of accretion onto a stellar magnetic field which additionally suggests that the open field lines may themselves carry much of the accretion torque (von Rekowski \& Brandenburg 2004; Long et al. 2007). So how much of the stellar field is in the form of open field? One clue to this may come from the detection of rotational modulation of X-ray emission from the COUP stars (Flaccomio et al. 2005). This suggests that the X-ray coronae are compact, with discrete bright and dark regions. Gregory et al. (2006a) have modelled the structure of $\mathrm{T}$ Tauri coronae and found significant rotational modulation, with X-ray periods that are typically equal to the optical period (or one half of it). The dark regions are sites of open field where the stellar wind escapes. Some preliminary measurements of such wind properties are now being made.

At the same time as observations of stellar magnetic fields have suggested a complex structure, modelling of accretion inflows and wind outflows have also raised questions about the applicability of "disk locking" models. In the original theory of disk locking a dipolar magnetic field connects to the disk at the truncation radius (Ghosh \& Lamb 1978; 


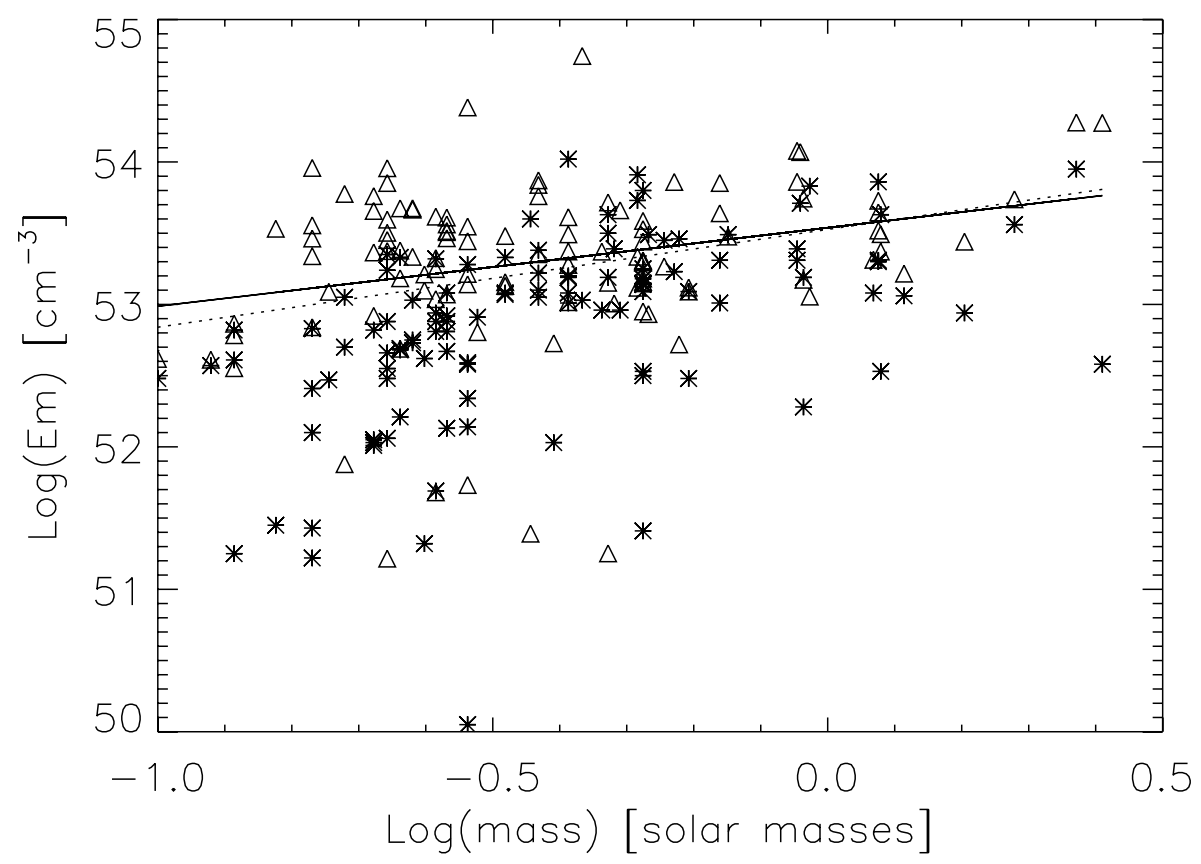

Figure 4. Emission measure as a function of stellar mass for stars in the COUP sample. Crosses show the observed values, while triangles show the calculated values. The solid line is the best fit to the calculated values, while the dotted line is the best fit to the observed values. Taken from Jardine et al. (2006).

Königl 1991). Strong fields are however needed in order to ensure that the truncation radius is close to the co-rotation radius, thus ensuring that the star is close to torque balance. This raises problems for the model since field strengths derived from circular polarisation measurements are of order $100 \mathrm{G}$, not the $1000 \mathrm{G}$ values needed by disklocking theory. In addition, the field lines that connect beyond the co-rotation radius and provide the spin down torque will be opened up by the shearing effect of the Keplerian velocity of the disk, and so will be unable to exert a torque on the star. The $\mathrm{X}$-wind model Shu et al. (1994) provides a mechanism for allowing accretion to occur whilst the angular momentum of the accreting material is removed from the system by a wind launched from a region close to the corotation radius. Observations of $\mathrm{T}$ Tauri jets however suggest they carry much more angular momentum than is present at this radius. Such a large amount of angular momentum must come from further out in the disk in the form of a disk wind.

An accretion powered stellar wind on the other hand carries away the angular momentum imparted to the star by the accretion process. Its effectiveness (relative to a disk wind) lies in the large lever arm that is associated with its large Alfvén radius. This wind is powered by the energy of the accretion flow itself. Indeed, a significant fraction of this energy is needed to drive the wind and a consistent model for this energy transfer process remains the greatest challenge for this theory. Observational signatures of such hot winds are now becoming accessible (Dupree et al. 2005). A very promising technique for examining the nature of such winds is the use of co-ordinated observations, using both FUSE data and Zeeman-Doppler imaging to determine the magnetic field structure. This has been done for AB Dor, a star in the post $\mathrm{T}$ Tauri phase which no longer 

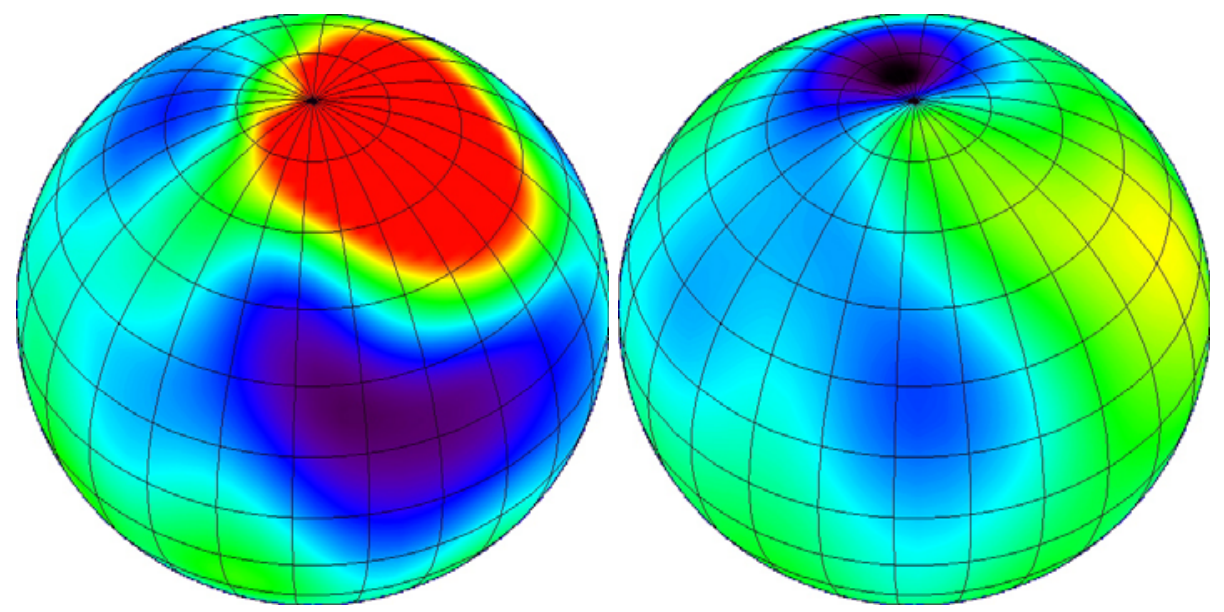

Figure 5. Surface radial field (left) and azimuthal field (right) for V2129 Oph. Blue shows negative polarity field, red shows positive polarity field (Donati et al. 2007).

has a disk, but whose field structure has been well studied. Field extrapolations from Zeeman-Doppler images show two dominant regions of open field in the upper hemisphere that are separated by about 180 degrees in longitude. UV spectra obtained with FUSE show variations with rotational phase that are consistent with this magnetic structure. Longitudes with open field regions show narrower, weaker OVI profiles whose asymmetry suggests outflows, while longitudes with mainly closed field regions show higher flux and the presence of compact active regions at mid-latitudes and of height 1.3-1.4 stellar radii (Dupree et al. 2006).

\section{What can we learn from time-dependent variations?}

While this is a promising technique for studying the structure of both the wind regions and the X-ray bright regions of $\mathrm{T}$ Tauri coronae, it provides only an instantaneous view of the system. This is a limitation shared by many $\mathrm{T}$ Tauri observations. A complementary approach is the type of long-term study that has been undertaken of AA Tau, which accretes at a moderate rate of only $10^{-9} \mathrm{M}_{\odot} \mathrm{yr}^{-1}$. Over the last 10 years there have been four campaigns monitoring the photometric and spectral diagnostics of magnetospheric accretion on timescales of days to years (for a review see Bouvier et al. 2007). The system is seen at a high inclination of about 75 degrees and the photometric brightness variations are consistent with a warp of the inner disk that is situated at $8.8 R_{\star}$ (approximately at the co-rotation radius). This warp may be caused by a large-scale magnetic field inclined to the stellar rotation axis (Romanova et al. 2003; Long et al. 2007), and is consistent with He I observations (Valenti \& Johns-Krull 2004). For AA Tau, H $\beta$ line profiles also show redshifted absorption components that appear near the middle of photometric eclipse, suggesting a spatial relationship between the disk warp and the funnel flows. The general characteristics of this variability are reproduced by non-symmetric radiative transfer models (Symington et al. 2005) although the predicted degree of variability is too high. On a timescale of weeks the change in the redshifted absorption component of $\mathrm{H} \alpha$ can be interpreted as due to field inflation (Lynden-Bell \& Boily 1994; Goodson et al. 1997) as field lines that link to the disk are sheared by the Keplerian flow until they expand and ultimately reconnect. On longer timescales, however, AA Tau's V-band eclipses change shape over several years, perhaps suggesting that the inner disk warp 


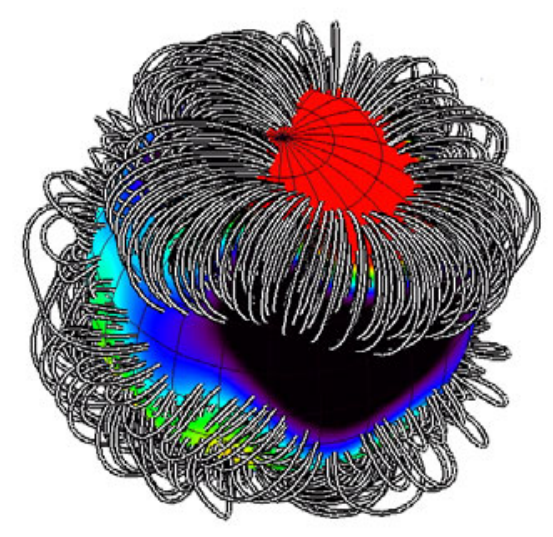

Figure 6. Calculated coronal field extrapolated from the surface radial field map of V2129 Oph shown in Fig. 5. The smaller field lines have been selected to show the field structure near the stellar surface.

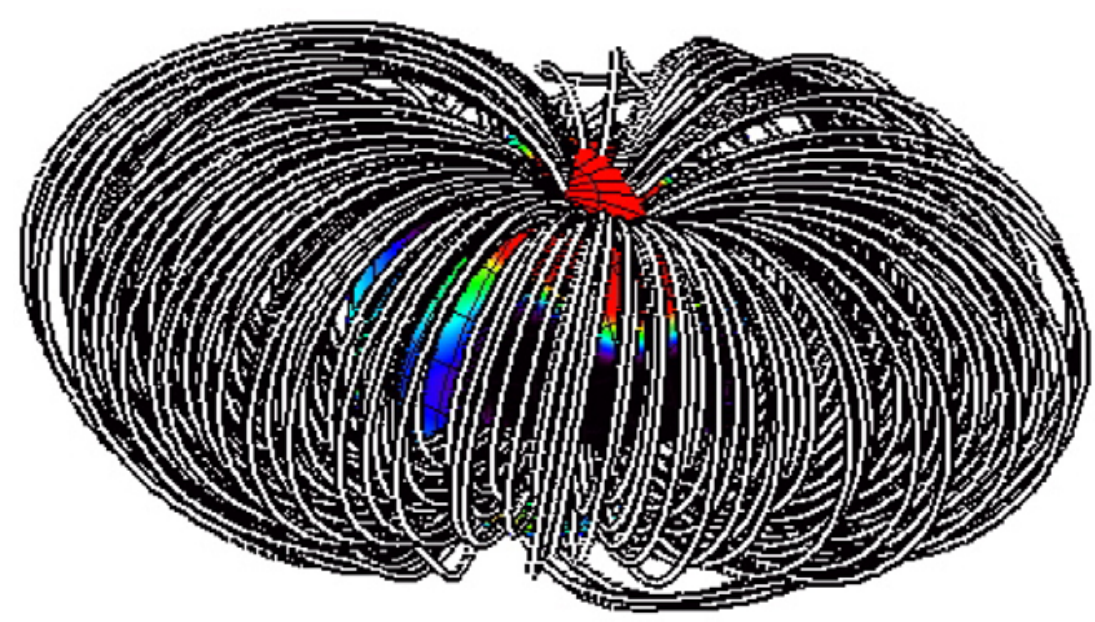

Figure 7. Calculated coronal field extrapolated from the surface radial field map of V2129 Oph shown in Fig. 5. The larger field lines have been selected to show the global structure.

changes on that timescale. This may be pointing towards a magnetic cycle, or longer timescale variation in the accretion process.

Perhaps one of the most promising approaches to studying the variable accretion processes in T Tauri stars is the use of multi-wavelength campaigns. This has been done for the Coronet cluster using simultaneous X-ray, radio, near-infrared and optical monitoring (Forbrich et al. 2007). This is the first attempt to examine the correlation between radio and $\mathrm{X}$-ray variability in class 0 and class 1 protostars. Preliminary results suggest that the variability in the radio and X-ray wavebands is uncorrelated, but that a relationship does exist between the luminosity at X-ray and radio wavelengths (Benz \& Guedel 1994).

In addition to the ubiquitous flaring-like variability seen in the X-ray emission of the COUP stars, there is also a clear rotational modulation in a small but significant number of these stars. Such modulation has previously been difficult to detect because it requires continuous observation over several rotation periods (Güdel et al. 1995; Marino 


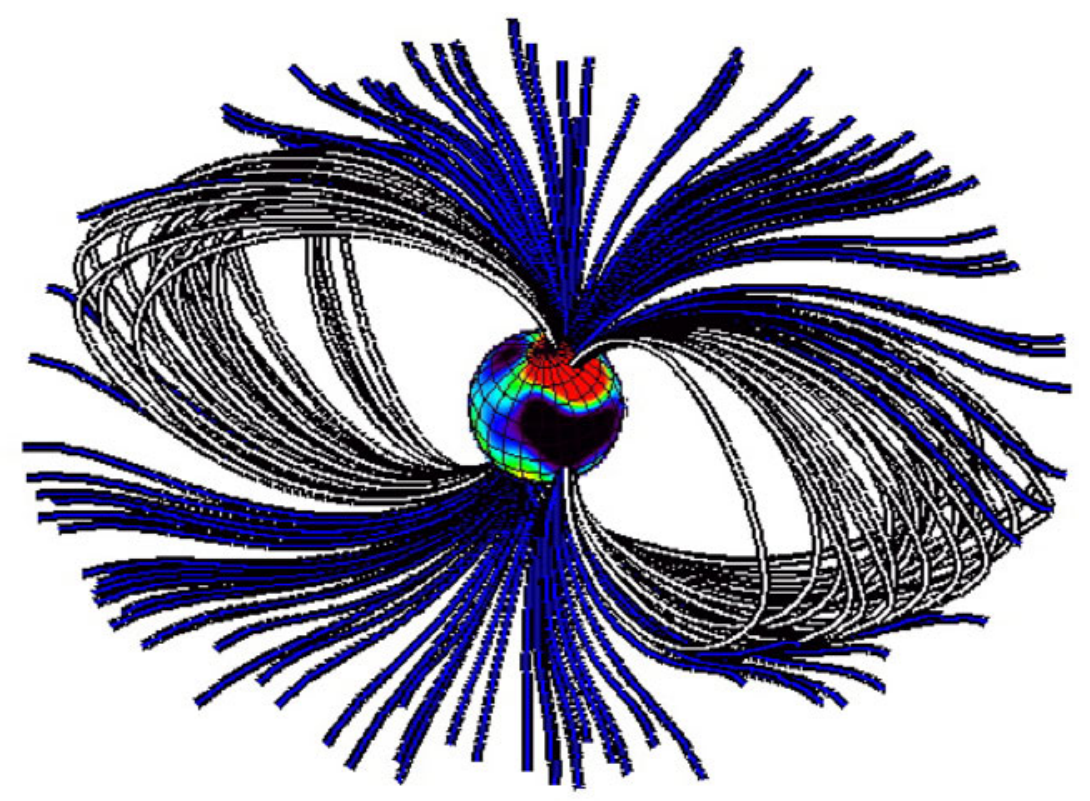

Figure 8. Some sample field lines that pass through the accretion disk inside the co-rotation radius and so are possibly capable of carrying an accretion flow. White denotes closed field lines, while blue denotes open field lines. We note, however, that these closed field lines would not be capable of confining the $\sim 10^{7} \mathrm{~K}$ plasma of the stellar corona and so are unlikely to be stable.

et al. 2003; Hussain et al. 2005). This rotational modulation shows clearly that although these stars appear to lie in the "saturated" part of the rotation-activity relation, they have coronae in which the dominant X-ray emission structures must be compact and cannot be distributed homogenously in longitude. Both in the COUP study and also more recently in the DROXO (Deep Rho Ophiuchi XMM-Newton Observation) study, there are flares for which modelling suggests the loops responsible must be large - perhaps reaching out into the accretion disk (Favata et al. 2005). If this represents a single flaring loop (rather than many loops) it suggests that the magnetic interaction between the star and the disk may be a violent one. Most flares from $\mathrm{T}$ Tauri stars are at much lower energies however and show a distribution in energy that is broadly compatible with what is observed on the Sun, which is consistent with energy release from a corona dominated by nanoflares (Caramazza et al. 2007).

The question still remains however of the contribution to the X-ray emission that may be made by the accretion process itself. Material falling onto a $\mathrm{T}$ Tauri star at the freefall speed $v_{f f}$ will be shocked to a temperature $T \propto v_{f f}^{2} \propto M_{\star} / R_{\star}$. For typical values, this gives $\mathrm{T}=2-4 \mathrm{MK}$ which would contribute to the soft X-ray spectrum. A few well studied stars such as TW Hya (Stelzer \& Schmitt 2004; Kastner et al. 2002) and more recently MP Muscae do indeed show the high densities and soft excess that would be expected from accretion, although this is not true of all $\mathrm{T}$ Tauri stars (Argiroffi et al. 2007). Of course not all of the infall will occur at the free-fall speed (as is apparent from modelling of accretion infalls (Gregory et al. 2006b) and there may be some absorption by the accretion column itself (Gregory et al. 2007). Modelling of the X-ray emission from the shock promises to help untangle the different contributions to the overall X-ray spectrum (Günther et al. 2007). 


\section{Zeeman-doppler imaging of $\mathbf{T}$ Tauri stars}

Our understanding of the nature of the magnetic field that links T Tauri stars to their disks has now entered a new phase with the availability of surface magnetic maps obtained using Zeeman-Doppler imaging. The first of these stars to be observed in this way is V2129 Oph, a young, moderately accreting T Tauri star aged around 1.5 - 3.0 My (Donati et al. 2007). With a derived period of $6.5 \mathrm{~d}$, a mass of $1.35 \pm 0.15 \mathrm{M}_{\odot}$ and radius of $2.4 \pm 0.3 \mathrm{R}_{\odot}$ it is fairly typical of many stars of this class. A map of the surface brightness shows a clear polar spot, in addition to many lower-latitude spots. Maps of the surface magnetic field derived from the photospheric lines show a complex topology with many regions of mixed polarity. Maps produced using the accretion-sensitive Ca IR triplet or HeI $5876 \AA$ line show a simpler structure with one dominant region of positive polarity at the same rotation phase as the greatest emission in the HeI line. This suggests that the coronal field at low to moderate heights is likely to be complex, while the largerscale field is much simpler. It is this larger-scale field that interacts with the disk and so is responsible for the single dominant region of accretion in the visible hemisphere.

Fig. 5 shows the surface radial and azimuthal magnetic fields. The strongest component of the field is a $1.2 \mathrm{kG}$ (tilted) octupole, while the dipole component is much weaker with a strength of only $0.35 \mathrm{kG}$. This can be seen most clearly by extrapolating the field and selecting out field lines of different heights. Fig. 6 shows the octupolar structure clearly, while Fig. 7 shows the tilted dipole. It is this dipolar field that survives out to the corotation radius and so is likely to interact with the disk. Thus while the closed-field corona (which will be bright in X-rays) is highly structured, the accreting field (shown in Fig. 8 ) is much simpler, in accordance with the behaviour of the HeI line. It seems, therefore, from this first example that the physical locations of the X-ray bright coronal regions and the accreting regions are quite distinct. Given the complexity of the small-scale field, it would be unlikely that a correlation would be found between the rotationally-induced optical and X-ray emission variation (Stassun et al. 2007).

\section{Conclusions}

Our understanding of the processes by which T Tauri stars accrete material from their disks while remaining at moderate rotation rates has advanced enormously over the past few years. The old models of stars with strong dipolar fields that lock the star to its disk have been replaced by (or perhaps only challenged by) new and more dynamic models. In these pictures, the star and the disk are not in torque balance. The accretion flows, which form into discrete funnels, transport angular momentum onto the star. Through some as yet unknown mechanism this process of accretion allows the star to drive a powerful wind that carries away enough angular momentum to keep the star rotating slowly.

The picture is more complicated than we might have thought some years ago, but observations of both the X-ray structure of $\mathrm{T}$ Tauri coronae and also of the magnetic field structure point to a degree of complexity inconsistent with a dipolar field. The very recent Zeeman-Doppler maps of V2129 Oph show a field that is simple on large scales, leading to a single accretion funnel in the observable hemisphere, while displaying significant complexity on the smaller scales on which the X-ray bright regions will be organised. There are also distinct regions of open field (see Fig. 9) which will be capable of carrying a hot stellar wind. More studies of this type, exploring stars at a range of evolutionary stages and so with a range of internal structures, would be extremely valuable.

The complexity seen in such "snapshots" of stars are also seen in the variability of the accretion process which appears to change on timecales from hours to years. Multiwavelength studies promise to be enormously valuable in studying the relationship between 


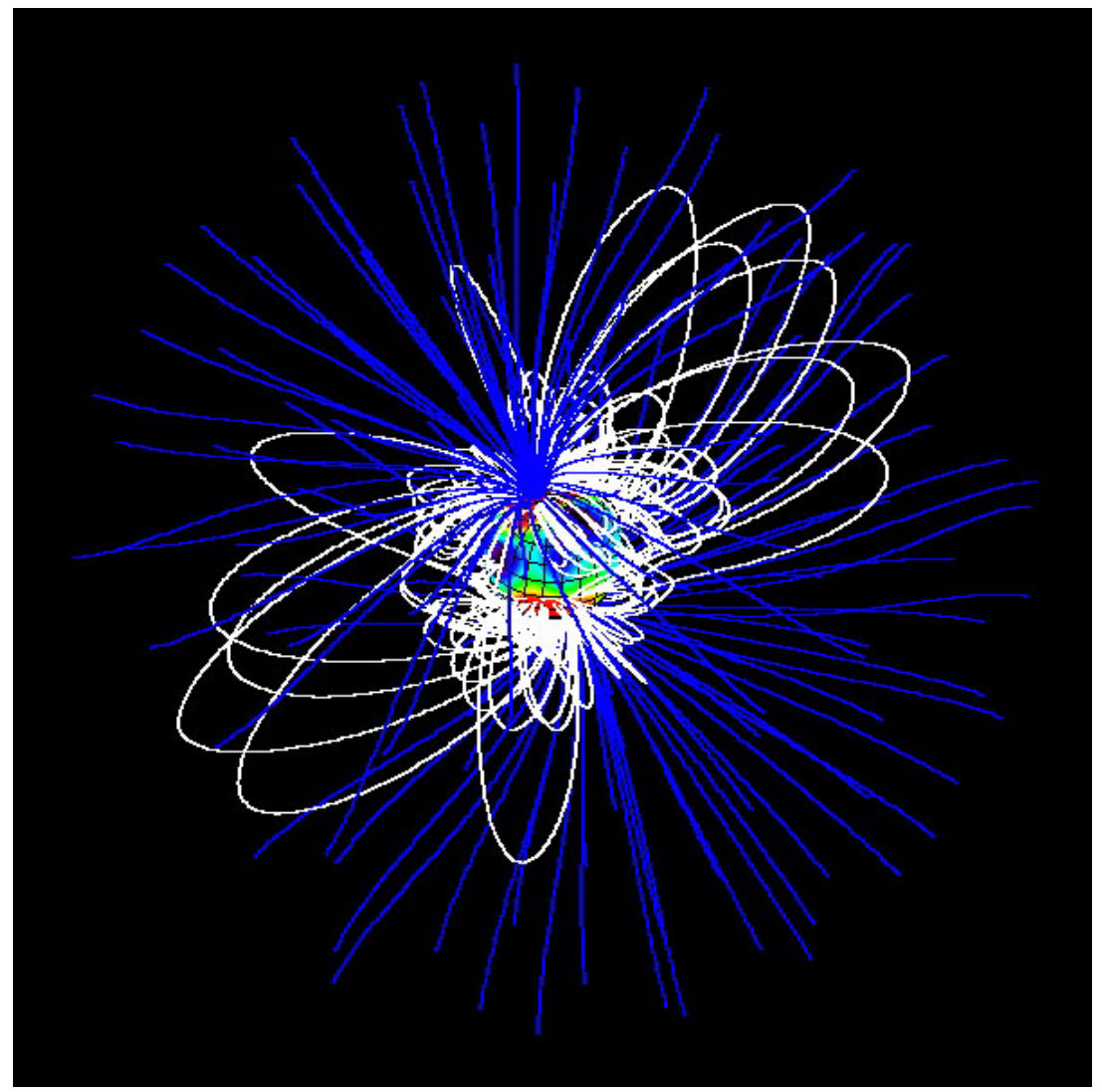

Figure 9. Calculated coronal field extrapolated from the surface radial field map of V2129 Oph shown in Fig. 5. White denotes closed field lines capable of confining hot, X-ray bright gas, while blue denotes open, wind-bearing field lines.

the closed-field, X-ray bright regions of the stellar corona, the accreting regions and the open-field wind-bearing regions. It appears that only by understanding the relationship between these different components of the coronal structure can we hope to understand the rotational evolution of $\mathrm{T}$ Tauri stars.

\section{References}

Altschuler, M. D. \& Newkirk Jr., G. 1969, Solar Phys., 9, 131

Argiroffi, C., Maggio, A., \& Peres, G. 2007, A\&A, 465, L5

Benz, A. O. \& Guedel, M. 1994, A\&A, 285, 621

Bouvier, J., Alencar, S. H. P., Harries, T. J., Johns-Krull, C. M., \& Romanova, M. M. 2007, in

Protostars and Planets V, ed. B. Reipurth, D. Jewitt, \& K. Keil, 479-494

Caramazza, M., Flaccomio, E., Micela, G., et al. 2007, ArXiv e-prints, 706

Donati, J.-F., Collier Cameron, A., Hussain, G., \& Semel, M. 1999, MNRAS, 302, 437

Donati, J.-F., Forveille, T., Cameron, A. C., et al. 2006, Science, 311, 633

Donati, J.-F., Jardine, M. M., Gregory, S., et al. 2007, MNRAS, submitted

Dupree, A. K., Ake, T. B., Brickhouse, N. S., Hussain, G. A. J., \& Jardine, M. 2006, in ASP

Conf. Ser. 348: Astrophysics in the Far Ultraviolet: Five Years of Discovery with FUSE,

ed. G. Sonneborn, H. W. Moos, \& B.-G. Andersson, 168-+

Dupree, A. K., Brickhouse, N. S., Smith, G. H., \& Strader, J. 2005, Ap. Lett., 625, L131

Favata, F., Flaccomio, E., Reale, F., et al. 2005, ApJS, 160, 469 
Flaccomio, E., Micela, G., Sciortino, S., et al. 2005, ApJS, 160, 450

Forbrich, J., Preibisch, T., Menten, K. M., et al. 2007, A\&A, 464, 1003

Getman, K. V., Flaccomio, E., Broos, P. S., et al. 2005, ApJS, 160, 319

Ghosh, P. \& Lamb, F. 1978, ApJ, 223, L83

Goodson, A. P., Winglee, R. M., \& Boehm, K.-H. 1997, ApJ, 489, 199

Gregory, S. G., Jardine, M., Cameron, A. C., \& Donati, J.-F. 2006a, MNRAS, 373, 827

Gregory, S. G., Jardine, M., Simpson, I., \& Donati, J.-F. 2006b, MNRAS, 371, 999

Gregory, S. G., Wood, K., \& Jardine, M. 2007, ArXiv e-prints, 704

Güdel, M., Schmitt, J., Benz, A., \& Elias II, N. 1995, A\&A, 301, 201

Günther, H. M., Schmitt, J. H. M. M., Robrade, J., \& Liefke, C. 2007, A\&A, 466, 1111

Hussain, G., Brickhouse, N., Dupree, A., et al. 2005, ApJ, 621, 999

Jardine, M., Cameron, A. C., Donati, J.-F., Gregory, S. G., \& Wood, K. 2006, MNRAS, 367, 917

Jardine, M., Collier Cameron, A., \& Donati, J.-F. 2002a, MNRAS, 333, 339

Jardine, M., Wood, K., Collier Cameron, A., Donati, J.-F., \& Mackay, D. H. 2002b, MNRAS, 336,1364

Johns-Krull, C. M., Valenti, J. A., \& Saar, S. H. 2004, ApJ, 617, 1204

Kastner, J. H., Huenemoerder, D. P., Schulz, N. S., Canizares, C. R., \& Weintraub, D. A. 2002, ApJ, 567, 434

Königl, A. 1991, ApJ, 370, L39

Long, M., Romanova, M. M., \& Lovelace, R. V. E. 2007, MNRAS, 374, 436

Lynden-Bell, D. \& Boily, C. 1994, MNRAS, 267, 146

Marino, A., Micela, G., Peres, G., \& Sciortino, S. 2003, A\&A, 407, L63

Preibisch, T., Kim, Y.-C., Favata, F., et al. 2005, ApJS, 160, 401

Rebull, L. M., Stauffer, J. R., Ramirez, S. V., et al. 2006, AJ, 131, 2934

Romanova, M. M. \& Lovelace, R. V. E. 2006, Ap. Lett., 645, L73

Romanova, M. M., Ustyugova, G. V., Koldoba, A. V., Wick, J. V., \& Lovelace, R. V. E. 2003, ApJ, 595, 1009

Shu, F., Najita, J., Ostriker, E., et al. 1994, ApJ, 429, 781

Stassun, K. G., van den Berg, M., \& Feigelson, E. 2007, ApJ, 660, 704

Stelzer, B. \& Schmitt, J. H. M. M. 2004, A\&A, 418, 687

Symington, N. H., Harries, T. J., Kurosawa, R., \& Naylor, T. 2005, MNRAS, 358, 977

Valenti, J. A. \& Johns-Krull, C. M. 2004, Ap\&SS, 292, 619

von Rekowski, B. \& Brandenburg, A. 2004, A\&A, 420, 17

Yang, H., Johns-Krull, C. M., \& Valenti, J. A. 2005, ApJ, 635, 466

Yang, H., Johns-Krull, C. M., \& Valenti, J. A. 2007, AJ, 133, 73

\section{Discussion}

REGEv: I don't think potential field models are appropriate for modelling T Tauri stars. Can you comment?

JARDINE: The only way to test a theory is to compare its prediction with observations. We are currently collaborating with Tim Harries to calculate the rotationally-modulated line profiles that would be predicted by our models and to compare these with the observed line profiles. The nature of this comparison will tell us if our field structures are largely consistent with the accretion indicators. 

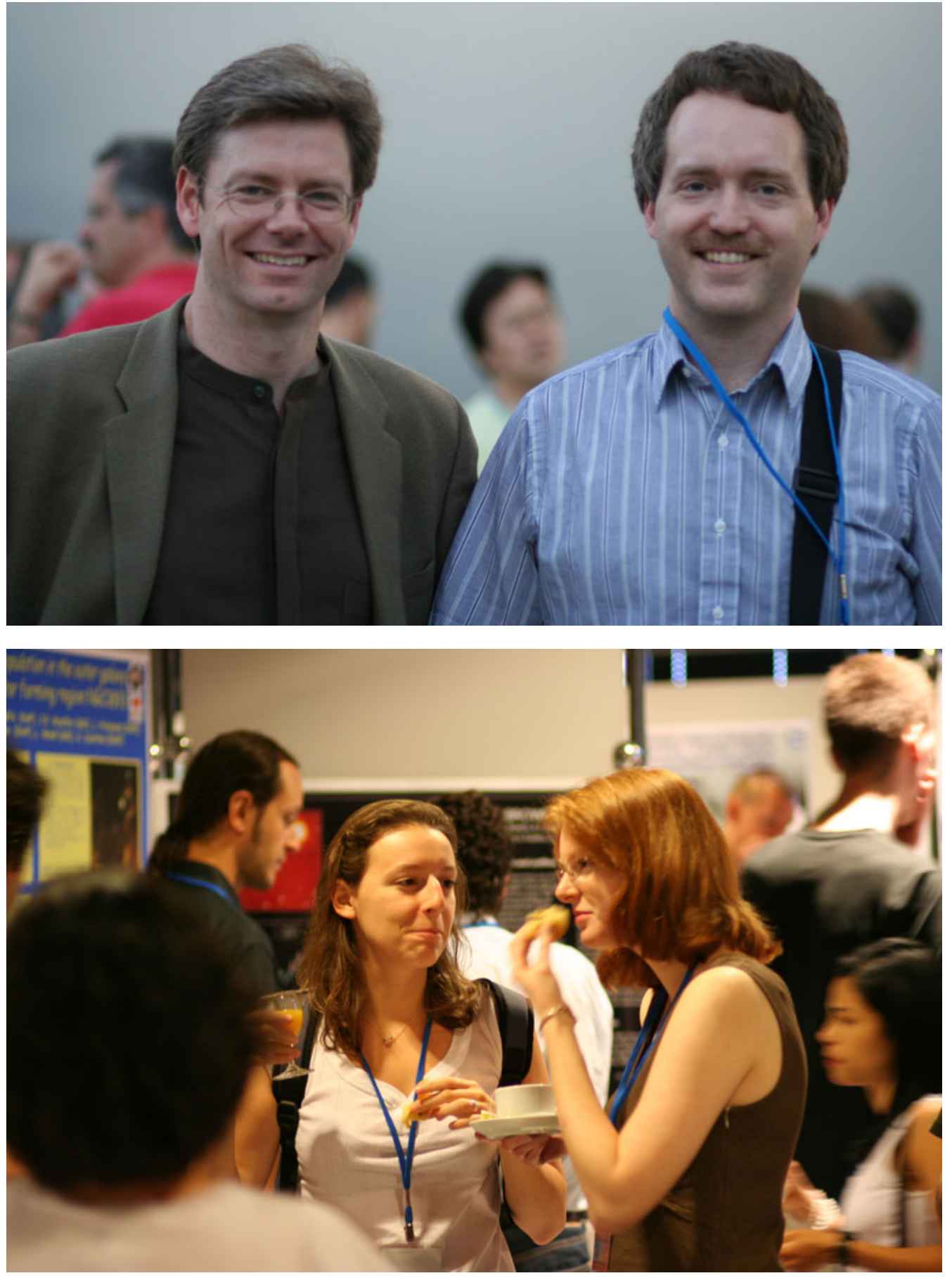